\title{
The shaker parameters estimation, a first step to virtual testing
}

\author{
Jonathan Martino ${ }^{1,}$, Kristof Harri $^{1}$ \\ ${ }^{1}$ Royal Military Academy, 30 Av. de la Renaissance, 1000 Bruxelles, Belgique
}

\begin{abstract}
The increase in computing resources makes simulation methods more affordable. The processing speed allows real time analysis or even more rapid tests analysis offering a real tool for test prediction and design process optimization. Vibration tests are no exception to this trend.

The so called 'Virtual Vibration Testing' offers solution among others to study the influence of specific loads such as optimized signals, to better anticipate the boundary conditions between the exciter and the structure under test or to study the influence of small changes in the structure under test.

This article will first present a virtual vibration test modeling with a main focus on the shaker model and will afterwards present the experimental parameters determination.

The classical way of modeling a shaker is to consider the shaker as a simple mechanical structure augmented by an electrical circuit that makes the shaker move. The developed model is a two degrees of freedom mechanical lumped parameters model and the electrical circuit model takes the coil impedance and the dynamic back-electromagnetic force into account. The establishment of the equations of this model, describing the dynamics of the shaker, is presented in this article and is strongly related to the internal physical quantities of the shaker. Those quantities will be reduced into global parameters which will be estimated through experiments in order to get a fully functional shaker model.

An experimental modal analysis will also be carried out to extract the modal parameters of the shaker and to combine them with the electrical measurements.

Finally this article will conclude with an experimental validation of the model.
\end{abstract}

\section{Introduction}

Vibration tests are an important part of the development process of a product and the optimization of these products in order to satisfy the imposed vibration requirements is essential. A constantly renewed aim is undoubtedly to push the limits of the possible. Is it possible to improve a product before it even exists? Is it possible to perform a physical test in less time or with less needed infrastructure? How to do more with less?

Virtual vibration testing aims to propose an approach to handle those aspects.

Increased computing capabilities make simulation tools more affordable. It is now possible to obtain a response in real time or even more rapidly from the response of a structure to a vibration test, to optimize the physical test or the structure to be tested, or to study the influence of specific loads, to implement the optimized signals in order to get more from the test infrastructure, to better anticipate the boundary conditions between the exciter and the structure under test, etc.

The simulation of a physical vibration test is carried out by modeling each of the constituent elements of this test $[1,2]$. The shaker plays a primordial role and the validity of the simulation depends almost exclusively on the validity of the model of the shaker. The latter is based on the physical characteristics of the constituent elements. A good determination of the parameters of the shaker will therefore be essential.

Figure 1 shows a cross-sectional view of an electrodynamic shaker. It essentially operates as a loudspeaker - vibration is generated by a current flowing in a coil immersed in a magnetic field - but where guides and suspensions have been added, making this system an electro-mechanical system.

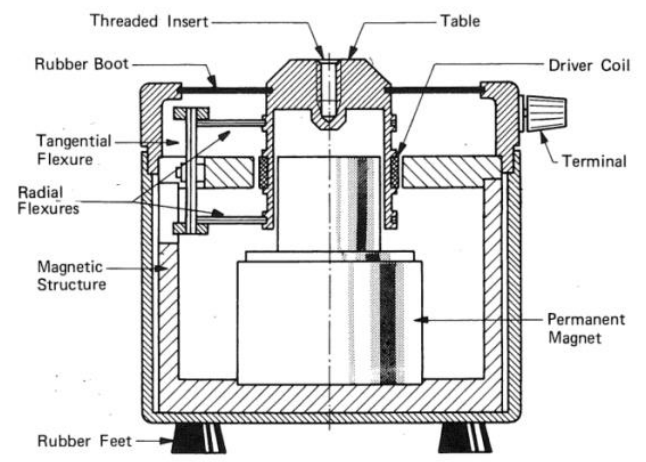

Fig. 1. Cross-sectional view of an electrodynamic shaker.

\footnotetext{
* Corresponding author: jonathan.martino@rma.ac.be
} 


\section{Shaker modeling}

The shaker can be modeled by mechanical lumped parameters but an electrodynamic relationship has to be added to represent the behavior of the coil when a signal is applied to its terminals.

Olsem [3] and McConnell [4] propose a model with two degrees of freedom (DOFs) while Lang and Snyder [5] propose a four DOFs model. Unlike Olsem and McConnel, this four DOFs model makes it possible to observe all the characteristic modes of a shaker isolation, suspension and coil mode.

Due to the small size of the shaker used in the work, the table and moving coil are considered as a rigid assembly [5]. The corresponding DOF can be eliminated and the model can be reduced to a three DOFs model (two mechanical and one electrical).

Figure 2(a) and Figure 2(b) respectively represent the mechanical and electrical models.

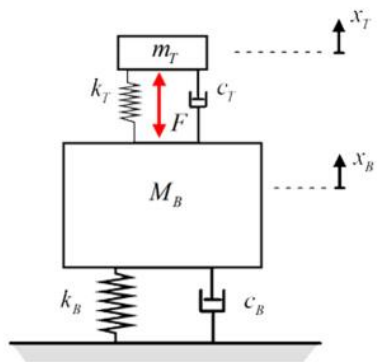

(a)

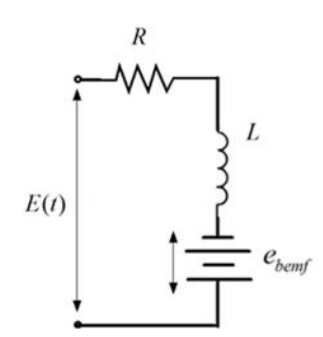

(b)
Fig. 2. Shaker model as a mechanical 2DOFs system (a) added with the electrical 1DOF model of the moving coil (b).

The force $\mathrm{F}$ applied to the table is generated by the coil and, by reaction, also applies to the body of the shaker. The coil moves vertically and its relative speed in the magnetic field creates a back electromagnetic force (bemf).

The integration of the equations of motion with the expression of the drive voltage as a function of the current flowing through the coil leads to the following equations [2]:

$$
\begin{gathered}
{\left[\begin{array}{ccc}
m_{T} & 0 & 0 \\
0 & M_{B} & 0 \\
0 & 0 & 0
\end{array}\right]\left[\begin{array}{c}
\ddot{x}_{T} \\
\ddot{x}_{B} \\
0
\end{array}\right]+\left[\begin{array}{ccc}
c_{T} & -c_{T} & 0 \\
-c_{T} & c_{B}+c_{T} & 0 \\
\mu_{b e m f} & -\mu_{b e m f} & L
\end{array}\right]\left[\begin{array}{c}
\dot{x}_{T} \\
\dot{x}_{B} \\
d i / d t
\end{array}\right]} \\
+\left[\begin{array}{ccc}
k_{T} & -k_{T} & -\mu_{f} \\
-k_{T} & k_{B}+k_{T} & \mu_{f} \\
0 & 0 & R
\end{array}\right]\left[\begin{array}{c}
x_{T} \\
x_{B} \\
i
\end{array}\right]=\left[\begin{array}{c}
0 \\
0 \\
E
\end{array}\right]
\end{gathered}
$$

Where $m_{T}, k_{T}, c_{T}, M_{B}, k_{B}$ and $c_{B}$ are the mass, stiffness and damping of the table and shaker body, respectively, and $R$ and $L$ are the electrical resistance and inductance of the moving coil. $\mu_{f}$ is the force constant and $\mu_{\text {bemf }}$ the back electromagnetic force constant of the system.

The left hand side of (1) describes the shaker behavior while the right hand side represents the drive voltage applied to the terminals of the shaker.

\section{Determination of the mechanical parameters}

The six mechanical parameters are the mass, stiffness and damping of the two mechanical degrees of freedom of the system, and are determined experimentally.

The method used consists in alternatively blocking each of the degrees of freedom and letting only the studied degree oscillate. We obtain two configurations: the shaker without isolation studs (free table test) to characterize the suspension of the coil, and the shaker with blocking of the vibrating table (blocked table test) to characterize the isolation studs.

The system is mounted on a larger shaker which is used to perform a sine sweep. The response of the system for the corresponding configuration is measured and identified with respect to the classical response of an oscillator with one degree of freedom.

\subsection{Experimental setup}

The shaker used is a modal shaker of type B\&K4809 whose mechanical characteristics supplied by the manufacturer are listed in Table 1.

In order to block the coil, we mounted a rigid structure between the body of the shaker and its vibrating table. It consists of a steel beam clamped at the top of the table and is depicted on Figure 3. We can notice the presence of an impedance head which will later be used in combination with a current sensor for the determination of the electrical parameters.

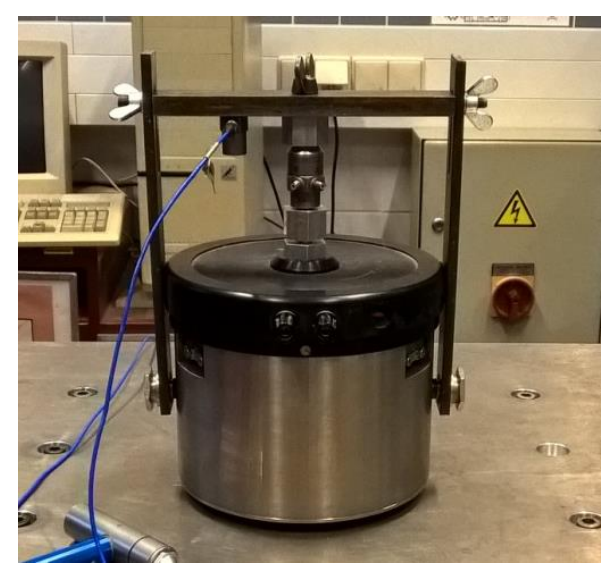

Fig. 3. The rigid structure limits the movement of the vibrating table. An impedance head is clamped between the vibrating table and the beam of the rigid structure.

We also have to eliminate the electrical degree of freedom by shorting the coil. During the free table test, the moving coil will have a relative speed with respect to the magnetic field. According the Lorentz force, a current tends to settle in the coil, but the latter can only circulate if the coil is short-circuited otherwise it creates a voltage at the terminals which would result in an electromagnetic damping on the coil. 


\subsection{Mechanical parameters identification}

Figure 4 and Figure 5 show the plotted response curves as a least square estimation of the measurements for both blocked table and free table configurations.



Fig. 4. Response curve of the isolation studs and the one DOF estimation in the blocked table configuration. With $f_{n}$ the natural frequency and $\xi$ the damping ratio.

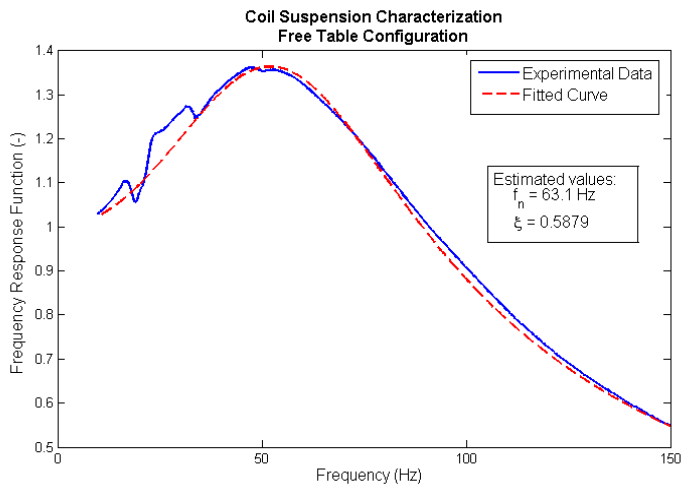

Fig. 5. Response curve of the vibrating table and the one DOF estimation in the free table configuration. With $\mathrm{f}_{\mathrm{n}}$ the natural frequency and $\xi$ the damping ratio.

The responses of the system correspond to $1 \mathrm{DOF}$ oscillators and using a least square estimation we obtained the values of the mechanical parameters. Those values are given in Table 1 with respect to the parameters given by the manufacturer.

\subsection{Modal parameters}

The developed method requires an external possibility of making the shaker vibrate and is quite difficult to apply to larger shakers. In this case, modal analysis is an alternative since the mechanical parameters and the modal ones are equivalent to characterize the system.

In this section, a short review of the modal theory will be presented to sufficiently master this equivalence as the relationship between movement, global coordinates, modal coordinates, and modal parameters.
Table 1. Mechanical parameters

\begin{tabular}{|l|c|l|l|}
\hline \multirow{2}{*}{ Parameter } & \multirow{2}{*}{ Symbol } & \multicolumn{2}{|c|}{ Values } \\
\cline { 3 - 4 } & & $\begin{array}{c}\text { Given by the } \\
\text { manufacturer[6] }\end{array}$ & $\begin{array}{c}\text { Determined } \\
\text { experimentally }\end{array}$ \\
\hline $\begin{array}{c}\text { Mass of the vibrating } \\
\text { table }\end{array}$ & $\mathrm{m}_{\mathrm{T}}$ & $60 \mathrm{~g}$ & $76 \mathrm{~g}^{\mathrm{a}}$ \\
\hline Mass of the body & $\mathrm{M}_{\mathrm{B}}$ & $8.3 \mathrm{~kg}$ & $8.375 \mathrm{~kg}$ \\
\hline $\begin{array}{c}\text { Damping of the } \\
\text { suspension of the } \\
\text { coil }\end{array}$ & $\mathrm{c}_{\mathrm{T}}$ & - & $35.43 \mathrm{Ns} / \mathrm{m}$ \\
\hline $\begin{array}{c}\text { Damping of the } \\
\text { isolation studs }\end{array}$ & $\mathrm{c}_{\mathrm{B}}$ & - & $564.3 \mathrm{Ns} / \mathrm{m}$ \\
\hline $\begin{array}{c}\text { Stiffness of the } \\
\text { suspension of the } \\
\text { coil }\end{array}$ & $\mathrm{k}_{\mathrm{T}}$ & $12 \cdot 10^{3} \mathrm{~N} / \mathrm{m}$ & $11.95 \cdot 10^{3} \mathrm{~N} / \mathrm{m}$ \\
\hline $\begin{array}{c}\text { Stiffness of the } \\
\text { isolation studs }\end{array}$ & $\mathrm{k}_{\mathrm{B}}$ & - & $3.06 \cdot 10^{5} \mathrm{~N} / \mathrm{m}$ \\
\hline
\end{tabular}

${ }^{\text {a}}$ For testing purposes, the original vibrating table is equipped with an extra $16 \mathrm{~g}$ fastening bolt.

\subsubsection{Theoretical aspects}

If we only consider the purely mechanical system as depicted in Figure 2(a), the global equations (1) can be generalized by:

$$
M \dot{z}+C \dot{z}+K z=F
$$

Where $M, C$, and $K$ are respectively the mass, damping, and stiffness matrices of the system.

Solving the eigenvalues problem for the free undamped system yields the transformation matrix $P$ between the modal coordinates $q$ and the global coordinates $z$ according [7]:

$$
z=P q
$$

The premultiplication by $P^{T}$ allows to diagonalize $M$ and $K$ since both are symmetrical. Despite that $C$ is also symmetrical, the damping matrix cannot be diagonalized and we can consider a linear combination of $M$ and $K$ [8]. Considering this, we now can express (2) as:

$$
m_{i} \ddot{q}_{i}+c_{i} \dot{q}_{i}+k_{i} q_{i}=Q_{i}(i=1,2)
$$

The modal parameters $m_{i}, c_{i}$, and $k_{i}$ are respectively the modal mass, damping and stiffness of the $i^{\text {th }}$ mode. $Q_{i}$ is the modal force and $q_{i}$ is the modal coordinate of the $i^{\text {th }}$ mode.

Whereas the system described by (2) is coupled, the system described by (4) is uncoupled since all the matrices have been diagonalized.

The solutions $q_{i}$ to the decoupled equations are given by:

$$
q_{i}=\frac{Q_{i}}{\left(k_{i}-m_{i} \omega^{2}+j c_{i} \omega\right)}=\frac{Q_{i}}{P k_{i}}(i=1,2)
$$

$P k_{i}$ is the characteristic polynomial of mode $i$ and $j$ is the complex constant. 
Using the matrix formulation, the real coordinate solution $z$ can be obtained from (3) and expressed by:

$$
z=\frac{P^{T} P}{P k} F=H_{i j} F
$$

With $F$ the global force vector representing the real forces applied in each DOF and $H_{i j}$ the dynamic influence coefficient matrix.

\subsubsection{Modal parameters calculation}

Using both mechanical equations (1) and values from Table 1, we are able to compute the modal parameters and the dynamic influence coefficient matrix. These functions are represented in Figure 6 for the undamped system and in Figure 7 for the damped system.


(a)ficient Matrix Damping
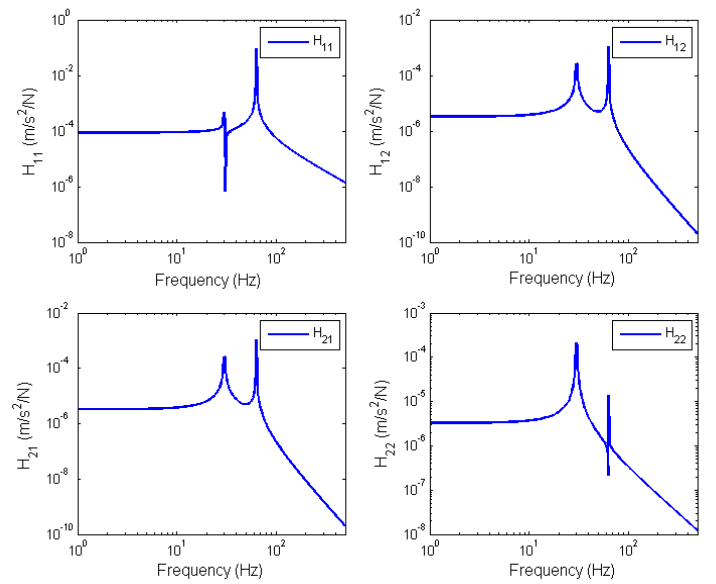

Fig. 6. Dynamic Influence Coefficient Matrix for the undamped system. The modal node 1 corresponds to the vibrating table whereas the modal node 2 corresponds to the body of the shaker.

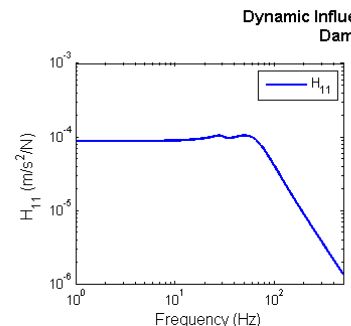

Coefficient Matrix

ped System
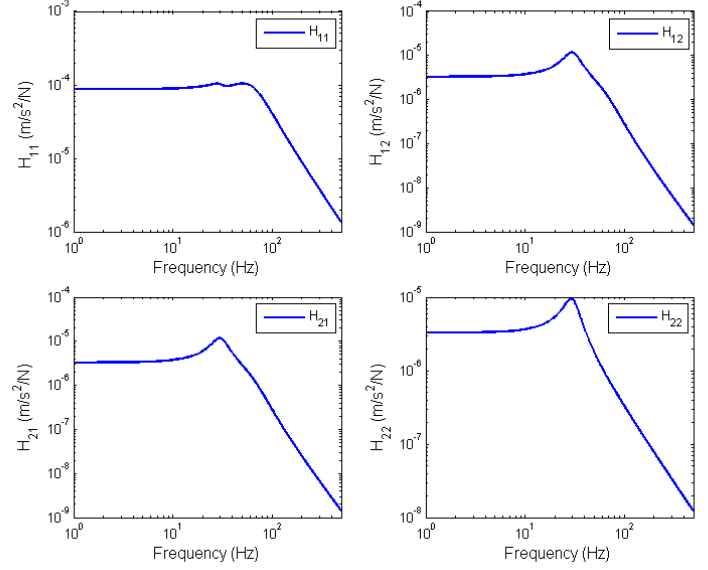

Fig. 7. Dynamic Influence Coefficient Matrix for the damped system. The modal node 1 corresponds to the vibrating table whereas the modal node 2 corresponds to the body of the shaker.
Figure 6 is clearer for mode detection than Figure 7 and shows two modes occurring at $30.2 \mathrm{~Hz}$ and $63.5 \mathrm{~Hz}$. This is consistent with the observed response showed in Fig 4 and 5.

The calculated modal parameters are given in Table2.

\subsubsection{Experimental modal analysis}

As said before, the calculation is only possible if we possess all the mechanical parameters but for larger shaker it is often impossible to block all the DOFs alternatively and to make it vibrate in order to extract the parameters. However, as presented in the theoretical review, the global mechanical parameters are equivalent to the modal ones.

In [9], Guillaume presented a method for the spectral identification of modal parameters by Least Squares Complex Frequency-Domain estimation. The LMS PolyMax method is based on this method and is recognized to be very fast and accurate for modal estimation [10].

We introduced a force with an impact hammer in the two DOFs of the shaker (respectively the table and the body) and measured the acceleration of those DOFs. The LMS PolyMax method computed the transfer functions and estimated the modal parameters with the stabilization diagram presented in Figure 8.

The corresponding values are given in Table 2 .

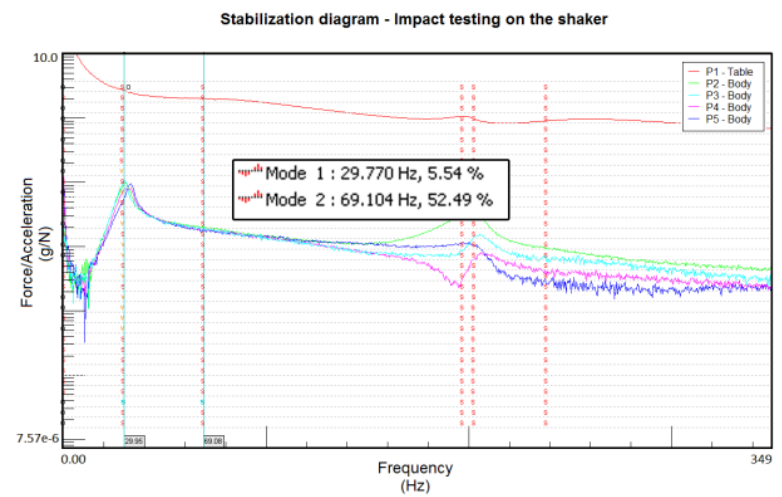

Fig. 8. Stabilization diagram of the impact testing on the shaker.

When looking at these values, we may observe that the experimental values match the calculated ones for the body-related parameters, especially for the modal mass and the modal stiffness, respectively $m_{2}$ and $k_{2}$, and in a less manner for the modal damping $c_{2}$. On the contrary, we have no match for the table-related parameters.

This difference is also noticeable in Figure 8. The response curves of the body (P2-P5) clearly show a resonance, making the body-related parameters identification possible, while the table resonance, identified in the stabilization diagram to be located at $69,104 \mathrm{~Hz}$, is not visible $(\mathrm{P} 1)$. We may conclude that the experimentally determined table-related parameters are not valid. 
This can be explained by the relative high damping in the suspension of the coil (see Figure 5) combined with the relative small force introduced into the shaker by the impact hammer, leading to a totally damped acceleration response of the table.

Table 2. Modal parameters

\begin{tabular}{|l|c|l|l|}
\hline \multirow{2}{*}{ Modal parameter } & \multirow{2}{*}{ Symbol } & \multicolumn{2}{|c|}{ Values } \\
\cline { 3 - 4 } & $\mathrm{m}_{1}$ & $77.2 \mathrm{~g}$ & $\begin{array}{c}\text { Determined } \\
\text { experimentally }\end{array}$ \\
\hline $\begin{array}{c}\text { Modal mass of the } \\
\text { vibrating table }\end{array}$ & $\mathrm{m}_{2}$ & $3.167 \mathrm{~kg}$ & $3.33 \mathrm{~kg}$ \\
\hline $\begin{array}{c}\text { Modal mass of the } \\
\text { body }\end{array}$ & $\mathrm{c}_{1}$ & $15.93 \mathrm{~kg} / \mathrm{s}$ & $1.9 \mathrm{~kg} / \mathrm{s}$ \\
\hline $\begin{array}{c}\text { Modal damping of the } \\
\text { suspension of the } \\
\text { coil }\end{array}$ & $\mathrm{c}_{2}$ & $196.15 \mathrm{~kg} / \mathrm{s}$ & $76 \mathrm{~kg} / \mathrm{s}$ \\
\hline $\begin{array}{c}\text { Modal damping of the } \\
\text { isolation studs }\end{array}$ & $\mathrm{k}_{1}$ & $\begin{array}{l}12.3 \cdot 10^{3} \\
\mathrm{~kg} / \mathrm{s}^{2}\end{array}$ & $\begin{array}{l}28.02 \cdot 10^{3} \\
\mathrm{~kg} / \mathrm{s}^{2}\end{array}$ \\
\hline $\begin{array}{c}\text { Modal stiffness of the } \\
\text { suspension of the } \\
\text { coil }\end{array}$ & $\mathrm{k}_{2}$ & $\begin{array}{l}1.143 \cdot 10^{5} \\
\mathrm{~kg} / \mathrm{s}^{2}\end{array}$ & $\begin{array}{l}1.15 \cdot 10^{5} \\
\mathrm{~kg} / \mathrm{s}^{2}\end{array}$ \\
\hline $\begin{array}{c}\text { Modal stiffness of the } \\
\text { isolation studs }\end{array}$ & \multicolumn{3}{|l}{} \\
\hline
\end{tabular}

\section{Determination of the electrical parameters}

Looking at (1) gives us the needed electrical parameters to be defined. We find $R$ and $L$, the resistance and the inductance of the coil, $\mu_{f}$, the force constant, and $\mu_{b e m f}$, the bemf (back electromagnetic force) constant of the system.

$R$ and $L$ are the intrinsic electrical properties of the coil and are purely passive. A simple measurement on the RLC meter will allow us to get those values. These are given in Table 3.

The quantities $\mu_{f}$ and $\mu_{\text {bemf }}$ are representative of the same phenomenon - the expression of the Lorentz force - but have neither the same causes nor the same effects.

Lorentz expresses that the charge carriers of a conductor immersed in a (non-collinear) magnetic field are subjected to a force when moving and this according to the following relation:

$$
\bar{F}_{q}=q(\bar{E}+(\bar{v} \times \bar{B}))
$$

With $F_{q}$ the force exerted on a charge carrier of electrical charge $q$ subjected to an electric field $E$, undergoing a speed $v$, and immersed in a magnetic field $B$.

The generalized expression of this force on a conductor of length $l$ is:

$$
F=B l i=\mu_{f} i
$$

The current $i$ flowing in the coil generates a force $F$ on the latter with respect to the force constant $\mu_{f}$.

The magnitude $\mu_{\text {bemf }}$ expresses the force undergone by the electrons in a conductor which moves in a magnetic field. The displacement of the coil generates an electric potential at the conductor terminals with respect to the bemf constant.

We study a system which on the one hand generates a force due to an applied current and on the other hand which creates an electrical potential with respect to the speed of the coil. This approach forms the basis of the development of a two-port network model and goes beyond the scope of this work. Different authors such as York, Smallwood or Guillaume develop this aspect deeper in detail [11-13].

\subsection{Experimental determination of the force constant}

First, we used the assembly already presented in Figure 3 which blocks the vibrating table. A voltage is applied to the terminals which generates a current, which creates a force. Due to the rigid structure, the table cannot move and no bemf can settle.

A measurement of the force with respect to the current yields the force constant. The latter is shown in Figure 9 for three different drive voltages.

We immediately notice a very strong structural response at $660 \mathrm{~Hz}$ due to the resonance of the structure originally intended to be rigid. By performing a modal analysis of this structure, we obtained its frequency response. The synthesized curve has been superimposed on the measured curves to emphasize the response due to the resonance of the beam structure.

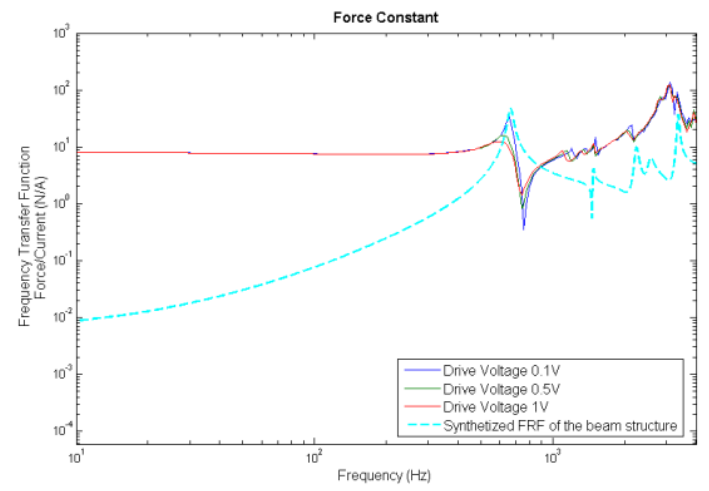

Fig. 9. Force Constant analysis for different drive voltages. The synthesized FRF of the beam structure is superimposed on the measured curves to emphasize the response due to the resonance of the beam structure.

Beyond this, the measurements tend to show an increase in damping with the drive voltage underlying a possible non-linearity of the system.

At this point any deduction is dangerous and should be avoided. Further analysis is required. Nevertheless, in the area below $100 \mathrm{~Hz}$, the relative theoretical error due to the resonating structure with respect to the unity does not exceed 3\%. Despite a relative variation in amplitude of $5 \%$ over the frequencies, the value of $\mu_{f}$ is considered to be constant. Its average value is $7.8493 \mathrm{~N} / \mathrm{A}$ against an initial value form the manufacturer of 6.4 N/A. These data are shown in Table 3. 


\subsection{Experimental determination of the bemf constant}

As we know the characteristics of the coil, we can build a test wherever the body of the shaker is fixed (to avoid measuring the influence of the isolation studs) but where the table is free to vibrate. The displacement speed of the table generates an electrical potential which counters the current and lets it decrease.

The electrical system is pure passive; the former current measurement allows us to determine the current loss. Taking as reference the current measured with the blocked table, the resonance of the beam structure will impose the same limitation.

Figure 10 illustrates the ratio between the bemf and the speed of the vibrating table. We conducted the test in two sets of added masses: $120 \mathrm{~g}$ and $502 \mathrm{~g}$.



Fig. 10. Bemf Constant analysis for different drive voltages and different added masses.

As we could expect, the curves are gathered by set but with a constant top 'quality factor'. The bemf is related to the speed of the coil, itself dependent on the driven mass. Next to this, we must keep in mind that the shaker also has its own response. Here we can clearly observe the influence of the response of the shaker. As the frequency response of the shaker is not constant, it will also not be the case for the bemf constant which will be a function of the frequency.

Table 3. Electrical parameters

\begin{tabular}{|l|c|l|l|}
\hline \multicolumn{1}{|c|}{$\begin{array}{c}\text { Electrical } \\
\text { parameter }\end{array}$} & Symbol & \multicolumn{2}{c|}{ Values } \\
\cline { 3 - 4 } $\begin{array}{c}\text { Resistance of the } \\
\text { coil }\end{array}$ & $\mathrm{R}$ & $\begin{array}{c}\text { Given by the } \\
\text { manufacturer }\end{array}$ & $\begin{array}{c}\text { Determined } \\
\text { experimentally }\end{array}$ \\
\hline $\begin{array}{c}\text { Inductance of the } \\
\text { coil }\end{array}$ & $\mathrm{L}$ & - & $1.28 \Omega$ \\
\hline Force constant & $\mu_{\mathrm{f}}$ & $\sim 6.4 \mathrm{~N} / \mathrm{A}$ & $7.8493 \mathrm{~N} / \mathrm{A}$ \\
\hline $\begin{array}{c}\text { Back } \\
\text { electromagnetic } \\
\text { force constant }\end{array}$ & $\mu_{\mathrm{bemf}}$ & - & $\begin{array}{l}\text { Frequency } \\
\text { varying }\end{array}$ \\
\hline
\end{tabular}

\section{State-space modeling and virtual testing}

\subsection{State-space model}

A State Space model allows a temporal approach of the simulation of a system. The equations governing the system must nevertheless be reformulated in order to correspond to the following formalism:

$$
\begin{aligned}
& \dot{x}=A x+B u \\
& y=C x+D u
\end{aligned}
$$

$x, y$ and $u$ are respectively the states vector, the outputs vector and the inputs vector. The four matrixes $A, B, C$, and $D$ characterize the system. It is in those matrixes that the mechanical en electrical parameters of the shaker will be introduced.

The shaker could be simulated based on a state space model derived from the general equations of motion (1) and computed using the 'lsim' function from Matlab.

On top of the simulation code, a test interface has been developed, managing the data (storing and generating) while the control algorithm manages the update process. A light routine splits the path into a physical acquisition or a simulated acquisition, sending and getting the signals to and from the shaker model instead of the real shaker. The data is then stored in an underlying layer of the interface and the process is totally transparent for the control algorithm.

This allows driving both physical testing and simulation over a same platform implementing and using the same control functions. This approach could offer a great correlation between physical and simulation results due to the fact that the difference mainly resides in the shaker modeling.

\subsection{Experimental results}

We will first retrieve the system's impulse response functions. A pulse of unit energy is introduced and the response is measured in each of the degrees of freedom. The responses of the body of the shaker and of the table are given in Figure 12 and Figure 13.

It can already be noted that the resonant frequency of the insulating pads is present. As for the table, it is not surprising to get this look. In fact, the input signal is a voltage applied to the terminals, which results in a force on the coil. The response on the table of a force introduced into the coil is the exact meaning of the transfer function $H_{11}$ presented in Figure 7 . These two curves are therefore very close.

In a second step, a 10 s sine sweep up to $2000 \mathrm{~Hz}$ is generated and introduced into the model. The Figure 11(a) and Figure 11(b) respectively show the temporal responses of the table and the body of the shaker. 

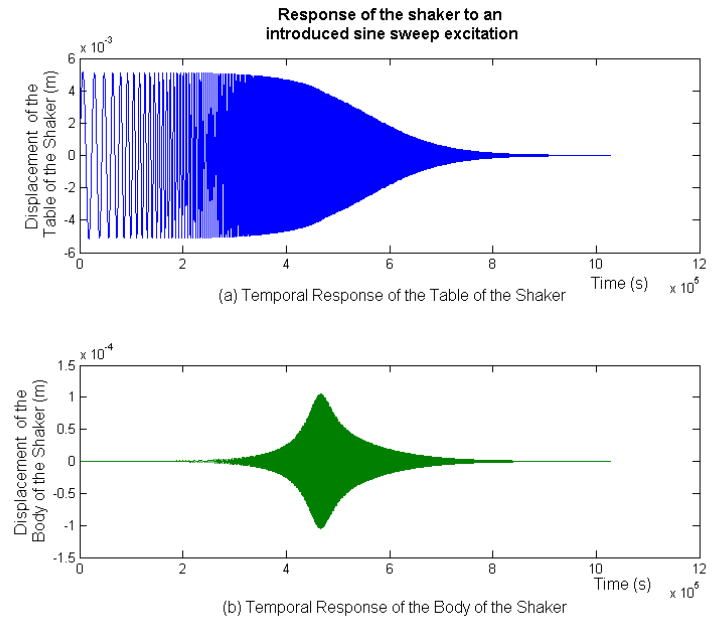

Fig. 11. Temporal response of the shaker to a sine sweep excitation. Response of the table (a) and response of the body (b).

We can see a very marked attenuation of the vibrations at the table, as well as a strong reaction of the body of the shaker at around $5 \mathrm{~s}$. We compute the frequency response function of those signals and superimpose them to the impulse response function of Figure 12 and Figure 13. We notice that the curves are very close to each other with a divergence above $2000 \mathrm{~Hz}$ due to the limitation of the sine sweep.

The simulation carried out makes it possible to obtain a temporal response to a virtual test and for which the replies characterize the system well.

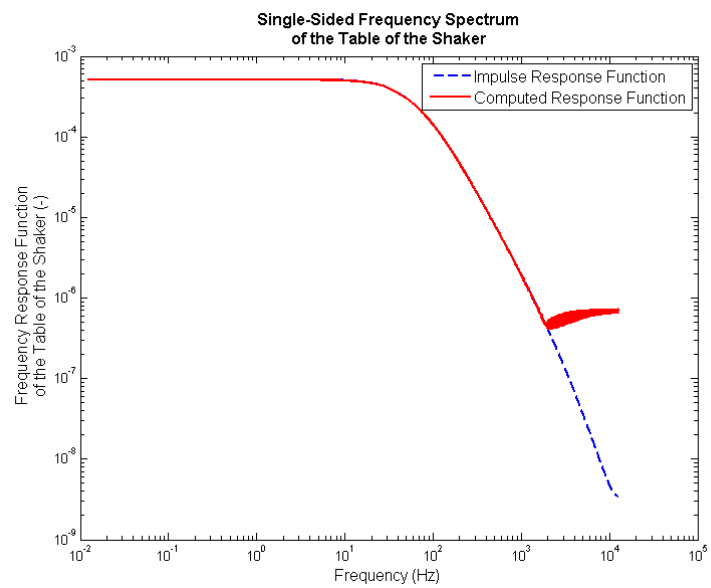

Fig. 12. Frequency Response Function and Impulse Response Function of the table of the shaker.

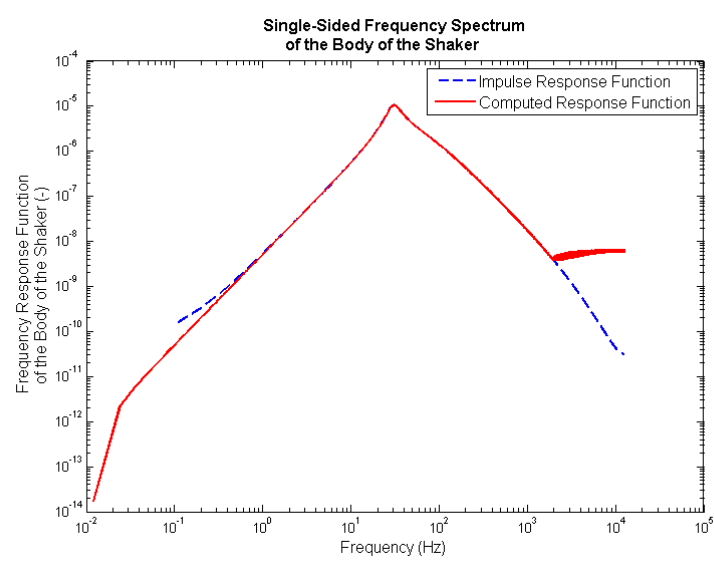

Fig. 13. Frequency Response Function and Impulse Response Function of the body of the shaker.

\section{Conclusions}

This article has highlighted an approach for determining the mechanical and electrical parameters of an electrodynamic shaker. Numerical and experimental modal analysis was also performed. The developed model has been successfully implemented in the linear state-space simulation environment for which the performed sine-sweeps illustrates the correlation with the experimentally observed behavior.

Nevertheless, many parameters presented in this work can be the object of a complementary research for their nonlinearity. Indeed, some parameters are expected constant what they are not at all. Some of these nonlinear effects have not been presented in this paper and are beyond the scope of this work.

The mass-dependence of the bemf constant as well as a pronounced interaction of the amplifier opens the way to a complementary analysis to this work. Interesting tracks would be the determination of the impedance matrix of the system as well as the modeling following a 2 port network.

\section{References}

1. S. Ricci, Proceedings of IMAC 27, (2009)

2. J. Martino, Proceedings of ISMA2016, (2016)

3. N.L. Olsem, Proceedings of the 4th International Modal Analysis Conference, 2, 1160-1167, (1986)

4. K.G. McConnell, Vibration Testing: Theory and Practice, (John Wiley \& Sons, NY, 1995)

5. G.F. Lang, D. Snyder, J. Sound Vib., 35(10), 24-33, (2001)

6. Brüel \& Kjaer, Product Data: Vibration Exciter Type 4809

7. D. Thorby, Structural Dynamics and Vibration in Practice, (Oxford: Elsevier, 127-129, 2008) 
8. A.G. Piersol, T.L. Paez, Harris' Shock and Vibration Handbook, (6th ed., New-York: McGraw-Hill, 21.12, 2010)

9. P. Guillaume, Proceedings of IMAC 21, (2003)

10. B. Peeters, G. Lowet, H. Van der Auweraer, J. Leuridan, J. Sound Vib., 38(1), 24-29, (2004)

11. R. Yorke, The Radio and Electronic Engineer, 39(4), 2, (1970)

12. D. Smallwood, Journal of the IES, 40(5), 2734, (1997)

13. P. Guillaume, R. Pintelon, J. Schoukens, IMTC91. Conference Record., 8th IEEE, 263-271, (1991) 